\title{
Sub-80 fs dissipative soliton large-mode-area fiber laser
}

\author{
Martin Baumgartl, ${ }^{1,2, *}$ Bülend Ortaç, ${ }^{1,3}$ Caroline Lecaplain, ${ }^{4}$ Ammar Hideur, ${ }^{4}$ \\ Jens Limpert, ${ }^{1,2}$ and Andreas Tünnermann ${ }^{1,2,5}$ \\ ${ }^{1}$ Friedrich-Schiller-University Jena, Institute of Applied Physics, Albert-Einstein-Strasse 15, 07745 Jena, Germany \\ ${ }^{2}$ Helmholtz-Institut Jena, Max-Wien-Platz 1, 07743 Jena, Germany \\ ${ }^{3} U N A M$-Institute of Materials Science and Nanotechnology, Bilkent University, \\ 06800 Bilkent, Ankara, Turkey (ortac@unam.bilkent.edu.tr) \\ ${ }^{4}$ CNRS UMR 6614 Complexe de Recherche Interprofessionnel en Aérothermochimie, Université de Rouen, \\ Avenue de l'Université BP 12, 76801 Saint Etienne du Rouvray Cedex, France \\ ${ }^{5}$ Fraunhofer Institute for Applied Optics and Precision Engineering, \\ Albert-Einstein-Strasse 7, 07745 Jena, Germany \\ ${ }^{*}$ Corresponding author: martin.baumgartl@uni-jena.de
}

Received March 31, 2010; revised June 4, 2010; accepted June 9, 2010;

posted June 18, 2010 (Doc. ID 126296); published June 30, 2010

\begin{abstract}
We report on high-energy ultrashort pulse generation from an all-normal-dispersion large-mode-area fiber laser by exploiting an efficient combination of nonlinear polarization evolution (NPE) and a semiconductor-based saturable absorber mode-locking mechanism. The watt-level laser directly emits chirped pulses with a duration of 1 ps and $163 \mathrm{~nJ}$ of pulse energy. These can be compressed to $77 \mathrm{fs}$, generating megawatt-level peak power. Intracavity dynamics are discussed by numerical simulation, and the intracavity pulse evolution reveals that NPE plays a key role in pulse shaping. (c) 2010 Optical Society of America

OCIS codes: $\quad 060.2310,060.2320,140.3510,140.4050,140.3615,140.7090$.
\end{abstract}

High-performance laser sources delivering ultrashort pulses with energies at the hundred nanojoule level or beyond will open up new directions for ultrafast scientific and industrial applications, ranging from highprecision laser structuring of various micro-/nanotargets to nonlinear optics. Rare-earth-doped fiber lasers present unique properties due to diffractionless propagation and the absence of thermo-optical problems. Because of the light confinement, fiber lasers offer a remarkable opportunity to develop highly stable laser sources, making them ideal candidates for real-world applications. The development of mode-locked fiber lasers operating in the normal dispersion regime to achieve higher pulse energies has been demonstrated [1,2]. Such lasers support dissipative solitons [3] and could produce sub-100 fs pulses with energies as high as $30 \mathrm{~nJ}$ using standard single-mode fibers [4]. More recently, exceptional performances in terms of pulse energy and peak power have been demonstrated in mode-locked fiber lasers using large-mode-area (LMA) photonic crystal fibers [믈] However, these lasers operate at a low accumulated nonlinear phase and produce relatively long pulses $>300$ fs.

The generation of ultrashort pulses in a dissipativesoliton laser strongly relies on the amount of nonlinearity accumulated along the cavity, which should be enough to ensure sufficient spectral broadening. However, generation of ultrashort pulses with broad bandwidths in an allnormal-dispersion laser needs a strong pulse-shaping mechanism to prevent excessive temporal expansion. One solution is the use of a narrow spectral filter to achieve self-consistent evolution inside the laser cavity [4]. Indeed, spectral filtering of a chirped pulse produces a strong amplitude modulation in the time domain, which increases with spectral breathing. Recently, the generation of sub-150 fs pulses with $24 \mathrm{~nm}$ spectral width has been demonstrated in an Yb-doped LMA fiber laser using a saturable absorber mirror (SAM) [10] .
In this Letter we report the generation of sub- $80 \mathrm{fs}$ pulses from a passively mode-locked all-normaldispersion laser featuring an LMA photonic crystal fiber. By exploiting the combined action of an SAM and nonlinear polarization evolution (NPE) for pulse shaping, the laser directly generates 1 ps chirped pulses with pulse energies above $160 \mathrm{~nJ}$ at watt-level average power. These pulses could be compressed down to 77 fs outside the cavity, reaching $\sim 1.2 \mathrm{MW}$ at the peak, which, to our knowledge, represents the highest peak power generated from a fiber oscillator. This performance competes with state-of-the-art femtosecond solid-state lasers. Numerical simulations show that pulse shaping in this laser is governed by the strong self-amplitude modulation induced by the SAM and NPE actions in combination with gain filtering.

The high-energy passively mode-locked fiber laser is set up in a sigma cavity configuration, as depicted in

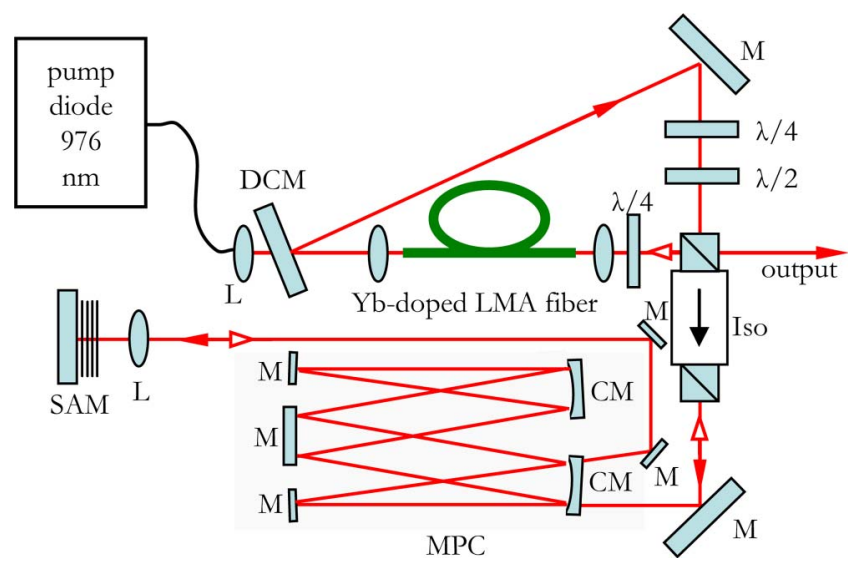

Fig. 1. (Color online) Schematic representation of the passively mode-locked low-repetition-rate Yb-doped LMA fiber laser: M, mirror; DCM, dichroic mirror; CM, curved mirror; L, lens; Iso, isolator; MPC, multipass cell; and SAM, saturable absorber mirror. 
Fig. 1. One key element is the $\mathrm{Yb}$-doped air-clad photonic crystal fiber with $40 \mu \mathrm{m}$ core and $170 \mu \mathrm{m}$ pump cladding diameter. The mode-field diameter of this fiber is about $30 \mu \mathrm{m}$. A fiber length of $1.3 \mathrm{~m}$ was chosen to obtain sufficient gain and low total cavity dispersion at the same time. Self-starting passive mode locking is achieved by employing an SAM in the linear part of the cavity. The SAM possesses a high modulation depth of $35 \%$, a saturation fluence of $20 \mu \mathrm{J} / \mathrm{cm}^{2}$, and a fast relaxation time of $\sim 600 \mathrm{fs}$. An optical isolator serves both as output coupler and as circulator to embed the linear part into the ring. A set of quarter- and half-wave plates is used to control the output coupling ratio and the NPE in the fiber. In order to achieve high pulse energies already at moderate average powers and, hence, limit the thermal load on the SAM, a confocal multipass cell (MPC) is implemented in the linear segment of the cavity. Therein, two curved mirrors with radii of $1.5 \mathrm{~m}$ reshape the beam at each round trip within the MPC.

By optimizing the saturation criteria for the saturable absorber in terms of spot size, stable mode-locked operation is obtained. Above the threshold, the laser delivers a single pulse train with a repetition rate of $6.15 \mathrm{MHz}$, which is monitored with a fast photodiode (200 ps rise time) and a $200 \mathrm{MHz}$ analog oscilloscope. The trace of the pulse train exhibits low amplitude noise behavior on all time scales of the scope (very similar to the case in Fig. 2 of [5]). Mode locking is initiated and stabilized by the SAM, however, to achieve stronger pulse shortening and, thus, considerable spectral broadening via self phase modulation (SPM); the wave plates were adjusted for significant contribution of NPE to pulse shaping. On the other hand, it should be mentioned that without the SAM, no mode locking was obtained at any position of the wave plates. With this optimization, the laser operates in a self-starting regime at a pump power of $6.2 \mathrm{~W}$, delivering chirped output pulses of only 1 ps duration at an average output power of $1 \mathrm{~W}$. At a pulse energy of $163 \mathrm{~nJ}$, the peak power can be estimated to be $\sim 150 \mathrm{~kW}$. This peak power is accessible without any postprocessing of the pulses. In contrast to [4], no additional narrowband spectral filter is needed to achieve

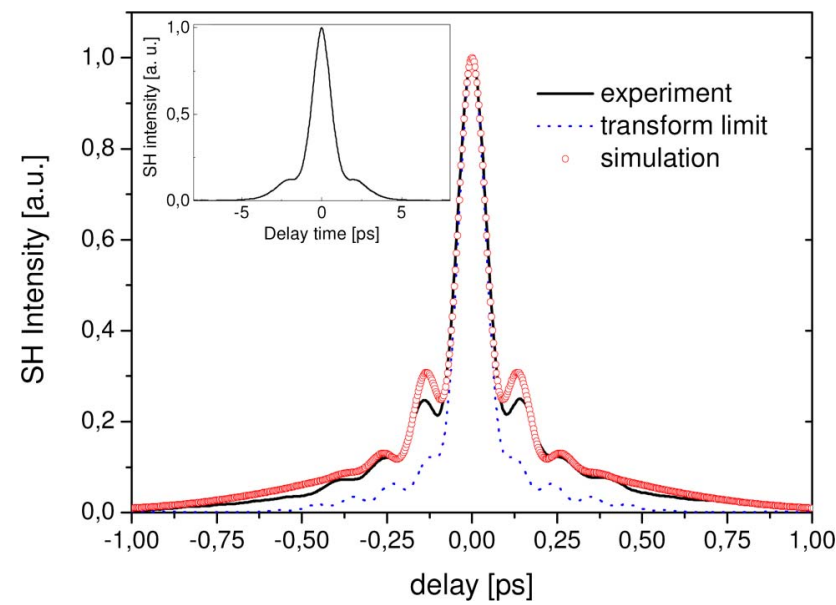

Fig. 2. (Color online) Autocorrelation traces of the output pulse (inset) and the dechirped pulse: solid curve, experiment; circles, simulation; dotted curve, transform limit calculated from experimental spectrum. stable mode locking. The combined action of NPE and SAM is sufficient for direct generation of high peakpower pulses with broadband spectrum at the laser output. The output pulse autocorrelation is shown in Fig. 2 (inset). It exhibits a pedestal revealing strong pulse wings. This confirms the strong contribution of the NPE mechanism for pulse shaping. Using a transmission grating pair with 1250 lines $/ \mathrm{mm}$, the output pulses could be compressed externally to the near-transform-limited duration of $77 \mathrm{fs}$. The autocorrelation trace of the compressed pulse is shown in Fig. 2 together with the autocorrelation of the transform-limited pulse, which was calculated from the power spectrum assuming a zero phase. The two traces show nearly the same width (FWHM), which indicates that the linear chirp is dominant. The optical spectrum (Fig. 3) exhibits a steep edged shape, which is typical for an all-normal-dispersion laser with strong SPM influence. As a result of these steep edges and a residual higher order phase, the dechirped pulses show small side lobes, causing a pedestal in the autocorrelation trace (Fig. 2). Taking the side lobes into account, the compressed pulses deliver a peak power of $\sim 1.2 \mathrm{MW}$, wherein the pulse energy is decreased to $130 \mathrm{~nJ}$ due to the compressor efficiency of $80 \%$. This is, to our knowledge, the highest peak power generated by a fiber oscillator.

Numerical simulations based on a nondistributed model, treating every part of the cavity separately by solving the nonlinear Schrödinger equation with a split-step algorithm, were carried out. Details on the numerical model are given in [11]. Absorption of the SAM is described by the rate equation model with a relaxation time of $600 \mathrm{fs}$ [12]. Suggesting that the NPE mechanism plays a key role in pulse shaping, an ideal saturable absorber with monotonically increasing transmission is introduced just after the gain fiber [11]. We note that our scalar model takes into account only the additional amplitude modulation provided by the NPE process and does not describe the polarization evolution inside the cavity. Besides the high-output coupling ratio at the NPE rejection port, additional losses of optical elements and fiber coupling are taken into account between the simulated cavity elements (Fig. 4). The experiment is well described by the model, as confirmed by comparison of the simulation

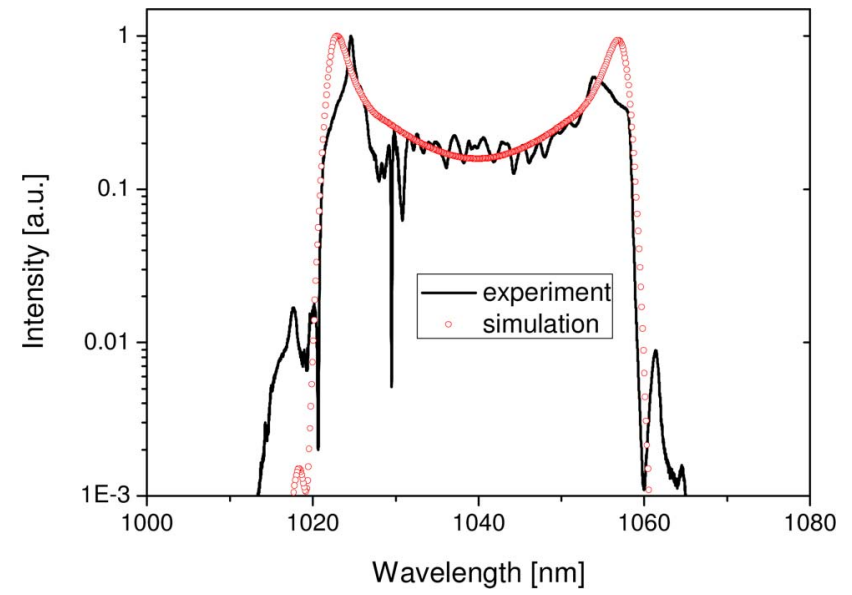

Fig. 3. (Color online) Spectrum of the output signal: solid curve, experiment; circles, simulation. 


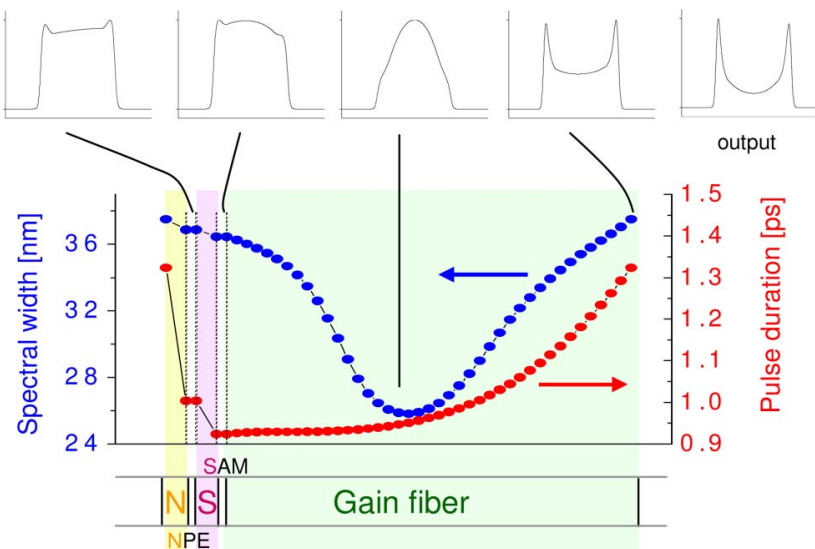

Fig. 4. (Color online) Intracavity evolution, spectral width (upper curve) and pulse duration (lower curve): top, spectral shape at different cavity positions; NPE, polarizer rejecting the pulse components rotated by nonlinear polarization evolution; and white sections between elements, passive loss in the cavity.

output with the experimental data in Figs. 2 and 3. The numerical result possesses steeper spectral flanks, which causes a slightly higher pedestal in the autocorrelation than is observed for the experimental case. The simulation (Fig. 4) shows that NPE is essential for pulse shaping. Temporal shortening is provided by both NPE and the SAM nonlinearity. It is well known that spectral filtering can substantially support self-consistent pulse evolution. Gain filtering significantly shortens the spectral width in the first part of the fiber, whereas the spectrum is broadened by Kerr nonlinearity in the second part. However, this laser shows new pulse evolution, as no additional filter element besides the gain bandwidth is needed. The broadband filter effect of the amplification process (as a result of gain spectrum and gain narrowing) together with the nonlinear temporal action of NPE and SAM is sufficient in our case to balance dispersive broadening. The spectral filtering does not contribute significantly to directly shorten the pulse in the time domain, as the temporal monotonic evolution in the gain segment shows. Low cavity dispersion together with high intracavity loss and strong amplification prevent excessive temporal broadening and enable self-consistent pulse evolution. When shifting the spectral filter action partly onto a passive filter element by inserting a broadband $(40 \mathrm{~nm})$ spectral filter in the simulation and increasing the gain bandwidth at the same time, agreement with the experimental results is reduced. The additional filter trims the pulse shape, hence, the minimum in spectral width shifts slightly toward the beginning of the fiber and significant SPM emerges earlier. Therefore the influence of dispersive broadening rises, which results in longer pulse durations at the output than are observed in the experiment. The evolution of the spectral shape along the cavity is shown in Fig. $\underline{4}$ (top). Spectral shaping is dominated by NPE, Kerr nonlinearity, and gain filtering. The first trims the edge peaks developed by the nonlinearity and forms a near-rectangular shape, whereas the latter leads to a parabolic-type shape in the middle of the gain fiber before strong nonlinear broadening at higher pulse energies sets in and forms the typical cat ears at the end. Both SAM and NPE have little influence on the spectral width. The nonlinear phase, which is accumulated during one cavity round trip, is about 1 order of magnitude higher than in [9], where an even larger core fiber was used in a purely SAM-mode-locked regime. The use of such a fiber would allow for straightforward scaling of the presented performance by a factor of more than five to multimegawatt peak power.

Summarizing, a passively mode-locked fiber laser is demonstrated that produces $163 \mathrm{~nJ}$ pulses with a pulse duration of $1 \mathrm{ps}$ at an average power of $1 \mathrm{~W}$ delivering a peak power of $150 \mathrm{~kW}$ without postprocessing. These pulses are compressed externally to a duration of $77 \mathrm{fs}$ at $130 \mathrm{~nJ}$, enabling peak powers of more than 1.2 MW. This performance is obtained by exploiting the combined action of an SAM and NPE together with an LMA photonic crystal fiber. Pulse-shaping mechanisms of the allnormal-dispersion laser are studied numerically and reveal a new pulse evolution without an additional spectral filter. To our knowledge, this is the first sub-80 fs dissipative soliton LMA PCF laser.

We acknowledge support by the Deutsche Forschungsgemeinschaft (DFG) (research unit 532) and the Inter Carnot \& Fraunhofer Program under project APUS.

\section{References}

1. A. Chong, W. Renninger, and F. Wise, Opt. Lett. 32, 2408 (2007).

2. J. An, D. Kim, J. Dawson, M. Messerly, and C. Barty, Opt. Lett. 32, 2010 (2007).

3. W. H. Renninger, A. Chong, and F. W. Wise, Phys. Rev. A 77, 023814 (2008).

4. K. Kieu, W. Renninger, A. Chong, and F. Wise, Opt. Lett. 34, 593 (2009).

5. B. Ortaç, J. Limpert, and A. Tünnermann, Opt. Lett. 32, 2149 (2007).

6. B. Ortaç, O. Schmidt, T. Schreiber, J. Limpert, A. Tünnermann, and A. Hideur, Opt. Express 15, 10725 (2007).

7. C. Lecaplain, C. Chédot, A. Hideur, B. Ortaç, and J. Limpert, Opt. Lett. 32, 2738 (2007).

8. Y.-J. Song, M.-L. Hu, C.-L. Wang, Z. Tian, Q. R. Xing, L. Chai, and C.-Y. Wang, IEEE Photon. Technol. Lett. 20, 1088 (2008).

9. B. Ortaç, M. Baumgartl, J. Limpert, and A. Tünnermann, Opt. Lett. 34, 1585 (2009).

10. C. Lecaplain, B. Ortaç, and A. Hideur, Opt. Lett. 34, 3731 (2009).

11. T. Schreiber, B. Ortaç, J. Limpert, and A. Tünnermann, Opt. Express 15, 8252 (2007).

12. N. N. Akhmediev, A. Ankiewicz, M. J. Lederer, and B. Luther-Davies, Opt. Lett. 23, 280 (1998). 